periungual peeling of fingers and elevated PLTs appeared. After the therapy administration, eosinophilia was observed . The periodic cardiological evaluation was negative.

Learning points/Discussion Features of KD are similar to those found in certain illnesses which are caused by toxin-producing bacteria such as scarlet fever.

Certain patients require more than one dose of IVIG before demonstrating an effect because there is a threshold level of $\operatorname{IgG}$ which is necessary to reduce the clinical signs of inflammation.

The identification of causative agents will result in the development of less expensive and more specific therapies.

\section{P397 MICROBIOLOGY OF GRAM-POSITIVE BLOODSTREAM INFECTIONS IN CHILDREN IN MINSK}

${ }^{1}$ Katsiaryna Serhiyenka*, 'Oksana Romanova, ${ }^{2}$ Aleksey Lazarev. ${ }^{1}$ Belarussian state medical university, Minsk, Belarus; ${ }^{2}$ City children's infectious clinical hospital, Minsk, Belarus

\subsection{6/archdischild-2019-epa.743}

The aim of this study was to investigate the role of gram-positive bacteria in the structure of pathogens isolated from blood cultures, to update later approaches to empirical therapy and to compare our data with current trends worldwide.

Material and methods In a retrospective study from 2009 to 2017, the etiological structure of identified blood flow infections was studied. The study was conducted in the viral and bacteriological laboratory of the City children's infectious clinical hospital in Minsk. Only for the period allocated 654 pathogen cultured from blood cultures 515 patients aged from 3 weeks to 18 years old. In patients with clinical signs of systemic infection (chills, tachycardia, hypotension, oliguria, impaired consciousness, behavior, rash, etc.) on the background of fever $38^{\circ} \mathrm{C}$ and above, blood was taken for microbiological examination. For the analysis of the etiological structure recorded of any pathogen isolated from blood cultures. A blood culture was considered positive when a single isolation of the pathogen. With the simultaneous or sequential release of several microorganisms belonging to different species, all strains were included in the analysis. The repeated selection of identical the causative agent in the statistical analysis of the pathogen fell only once. Hemocultures were isolated using a BacT/alerT 3D hemoculture analyzer using commercial culture media (BacT/alerT SA, BacT/alerT SN, biomérieux, France). Identification of microorganisms was carried out on the automatic microbiological analyzer Vitek 2 Compact (bioMérieux, France).

Results According to the results of the study, the proportion of gram-negative bacteria in the structure was $31.7 \%$ $(n=208)$, gram-positive bacteria $-61.9 \% \quad(n=404)$, fungi $6.4 \%(n=42)$. During the analyzed period, the predominance of gram-positive microorganisms in the structure of bacteremia remains.

The structure of gram-positive bacteria $(n=404)$ was dominated by staphylococci $(62.1 \%)$, with the most frequently isolated coagulase-negative types $(n=212 ; 84.5 \%)$. Among all staphylococci $(n=25.1)$, the most frequently isolated cases were S. epidermidis $-63.3 \%$, and S. aureus $-15.1 \%$. The spectrum of streptococci $(n=66)$ was as follows: Str. viridans groups (42.4\%), Str. pneumonia (27.3\%), Str. agalacia $(19.7 \%)$ and Str. pyogenes $(10.6 \%)$. Enterococci were isolated in 31 patients $(7.7 \%$ of cases), with almost the same frequency met Enterococcus faecalis and Enterococcus faecium $(41.9 \%$ and $38.7 \%$, respectively). During the study period, $7(1.7 \%)$ strains of Corynebacterium spp. were isolated. The data obtained by us fully correspond to modern studies from other countries, according to which the most common pathogens of blood flow infections are coagulase-negative staphylococci (especially epidermal).

\section{P398 A CASE OF NEONATAL PLASMODIUM VIVAX MALARIA CAUSED BY EXCHANGE TRANSFUSION IN A G6PD DEFICIENT BABY}

Muhammad Ayyaz ${ }^{*}$, Syed Muhammad Hassan, Yaseen Badar. Saidu Group of Teaching hospital, Swat, Pakistan

10.1136/archdischild-2019-epa.744

Neonatal malaria is one of the differential diagnosis of sepsis in malarial endemic areas. Neonatal malaria is often ignored and missed as it is considered to be very rare. Transfusion acquired malaria is among the causes of neonatal malaria, as blood is rarely screened for malarial parasite. Here we present a unique case of neonatal malaria caused by exchange transfusion, which was done for indirect hyperbilirubinemia in a G6PD deficient baby.

\section{P399 APPLICATION OF A RAPID STREP TEST IN THE IDENTIFICATION OF BETA HAEMOLYTIC STREPTOCOCCUS GR. A (STUDY CONDUCTED IN DRACEVO AND ITS SURROUNDING)}

${ }^{1}$ Svetlana Smugreska*, ${ }^{1}$ Mitka Trencheva, ${ }^{2}$ Velibor Tasic. ${ }^{1}$ private Paediatric Practice, Skopje, Macedonia, The Former Yugoslav Republic Of; ${ }^{2}$ children's Clinic -Nephrology Department, Skopje, Macedonia, The Former Yugoslav Republic Of

\subsection{6/archdischild-2019-epa.745}

Introduction Most common cause of the major upper respiratory infections is Beta haemolyticus streptococcus gr A. It can cause pharyngitis, tonsillitis, scarlet fever, otitis, sinusitis and it can trigger skin infections such as: impetigo, phlegmon, erysipelas. It has seldom been considered to be the cause of peritonsillary abscess, supravital meningitis, septic shock.

Objective Rapid strep test provides prompt diagnosis and implementation of appropriate therapy. Complications such as APSGN, as well as rheumatic fever, vasculitis etc. are preventable.

Methods and materials A study on sample of 380 children at the age of 3 to 13 years has been conducted. All of them have simultaneously undertaken a rapid strep test and a microbiological culture with a waiting period of 2 to 3 days. The research involves two groups of children: the first one engages 267 children with two parallel symptoms: sore throat and lymph glands; sore throat and abdominal pain, sore throat and neck pain, high temperature followed by headache etc.

The second group contains of 113 children, facing with tonsillitis, high temperature, Ly glandulae on neck, vomiting followed by abdominal pain. 